\title{
Erfahrungen und Ergebnisse aus der begleitenden Erhebung zum Pilot w-fFORTE Innovatorinnen
}

\author{
Autorinnen: Charlotte Alber (FFG), Laura Dusl (WPZ Research), Brigitte Ecker (WPZ Research), \\ Sabine Pohoryles-Drexel (BMDW)
}

Mit w-fFORTE Innovatorinnen strebt das Bundesministerium für Digitalisierung und Wirtschaftsstandort (BMDW) an, Frauen in standortrelevanter Forschung und Innovation (F\&l) gezielt zu unterstützen und sichtbar zu machen. Hochqualifizierte Frauen werden darin bestärkt, ihre Ideen zu entfalten, neue Netzwerk-Kontakte aufzubauen und zu mehr Gestaltungsspielräumen und beruflicher Weiterentwicklung zu gelangen. Die FFG hat dafür im Zeitraum 2018-2019 in enger Zusammenarbeit mit dem BMDW ein innovatives Leadership- \& Empowerment-Programm entwickelt. Der erste Durchgang fand als Pilot von Juni 2020 bis April 2021 mit insgesamt 21 Innovatorinnen statt, die von einer Jury aus einer Vielzahl von Bewerberinnen ausgewählt wurden. Während der Pilot-Laufzeit wurde eine begleitende Erhebung durch WPZ Research durchgeführt. Ziel war es, die Wirksamkeit des Pilots zu prüfen und Evidenzen für eine mögliche Weiterführung der Aktivitäten zu erhalten.

\section{Ist Frauenförderung noch zeitgemäß?}

Ganz in der Tradition von w-fFORTE - "Wirtschaftsimpulse von Frauen in Forschung und Technologie“ -, das evidenzbasiert und als "lernendes Programm" von Anfang an mittels Studien und (Begleit)evaluierungen ${ }^{1}$ passgenaue Formate entwickeln wollte, um Barrieren, die der Karriereentwicklung von Forscherinnen und Technikerinnen entgegenstehen, und Rahmenbedingungen, die sie an der vollen Entfaltung ihres Potenzial hindern, zu minimieren, wurde auch das Pilot-Format w-fFORTE Innovatorinnen unter Einbeziehung der Zielgruppe gestaltet. Anhand von qualitativen Interviews mit erfolgreichen Role Models aus F\&l, einer Fokusgruppe mit Leiterinnen von FFG-geförderten Projekten und mittels einer Online-Umfrage wurde versucht, besser zu verstehen, wie die beruflichen Wege von erfolgreichen Frauen im Themenbereich Forschung, Technologie und Innovation (FTI) verlaufen sind, was besonders förderlich dabei war und was Frauen im aktuellen FTIUmfeld benötigen, um ihr Potential für Wirtschaft und Gesellschaft möglichst uneingeschränkt zu entfalten.

Seit dem Jahr 2005 ist das Programm w-fFORTE Bestandteil der Frauenförderungsaktivitäten des BMDW und der FFG. Es wurde vor dem Hintergrund der Unterrepräsentanz von Frauen in FTI entwickelt und wendet sich in Ergänzung zu den Aktivitäten des BMBWF und des BMK (früher: BMVIT) an Forscherinnen und Innovatorinnen in der anwendungsorientierten und kooperativen Forschung. Mittels Veranstaltungen, Karriere-Trainings, Studien sowie der einmaligen, sehr erfolgreichen, Impulsförderaktion "Laura Bassi Centres of Expertise" (8 von Top-Forscherinnen geleitete transdisziplinäre Forschungskooperationen mit der Wirtschaft, Laufzeit: 2009-2018) wurden exzellente Forscherinnen darin unterstützt, ihren eigenen Weg zu gehen. Förderliche Rahmenbedingungen dafür wurden mit der differenzierten Ausgestaltung des Programms "Laura Bassi Centres of Expertise" ermöglicht. So wurde bspw. bei der Ausschreibung ein neues, zweistufiges Auswahlverfahren entwickelt und eingesetzt, das nicht nur die bisherigen wissenschaftlichen

\footnotetext{
${ }^{1}$ Siehe hierzu: Konzept für die Entwicklung von Maßnahmen des bmwa in fFORTE - fteval Repositorium; Konzeptive Vorbereitung der Einführung von Laura Bassi Centres of Expertise | ÖGUT (oegut.at); Begleitende Evaluierung der Impulsaktion "Laura Bassi Centres of Expertise": Erste Zwischenergebnisse - fteval Repositorium; Begleitende Evaluierung der Impulsaktion 'Laura Bassi Centres of Expertise' - fteval Repositorium; Fortschrittsbericht 2012: Begleitende Evaluierung der Impulsaktion „Laura Bassi Centres of Expertise“ - fteval Repositorium; Begleitende Evaluierung der Impulsaktion „Laura Bassi Centres of Expertise“ - fteval Repositorium
} 
Leistungen der Forscherinnen, sondern auch deren Zukunftspotenziale im Bereich Forschung wie auch in Management, Teamführung und Karriereplanung bewertete ${ }^{2}$.

Die Erkenntnisse aus dieser europaweit einzigartigen Initiative wurden nicht nur international positiv wahrgenommen, sie dienten auch als Inspiration für andere Förderprogramme in der FFG. Auch neue FFG-Förderformate, wie z.B. Laura Bassi 4.0 (Fokus: Digitalisierung und Chancengerechtigkeit), Ideen Lab 4.0 (Fokus: inter- und transdisziplinäre Zusammenarbeit und neues Auswahlverfahren mittels Sandpit-Methode) ${ }^{3}$ und die laufende Arbeit von w-fFORTE (z.B. durch spezielle Workshops zum Thema interdisziplinäre Zusammenarbeit, Arbeiten in gemischten Projekt-Teams) gehen auf Erkenntnisse der begleitenden Evaluierung der Laura Bassi Centres of Expertise zurück. Bei all diesen Entwicklungsarbeiten wurde zudem anhand aktueller Studien, Statistiken und eigener Erhebungen immer wieder hinterfragt, ob es auch weiterhin spezifische Frauenförderung braucht bzw. welche Aktivitäten notwendig sind, um zeitgemäß auf die Anforderungen in Forschung und Innovation zu reagieren.

In der Vorbereitung von w-fFORTE Innovatorinnen zeigte sich, dass es trotz zum Teil hoher bzw. steigender Absolventinnen-Zahlen an den Universitäten und Fachhochschulen noch immer relativ wenige Frauen in gestaltenden Rollen in der anwendungsorientierten Forschung bzw. als Unternehmerinnen, als Führungskräfte im Technologiebereich oder als Leiterinnen in drittmittelfinanzierten Projekten gibt. Spezifische Frauenförderungsaktivitäten sind weiterhin auch im Segment der hochqualifizierten Frauen notwendig. Aus den qualitativen Interviews wurde auch klar, dass als "ausschlaggebend für die Verwirklichung von Vorhaben“, neben den persönlichen Eigenschaften, das Thema Empowerment (im Sinne eines unterstützenden privaten und beruflichen Umfelds), Austauschmöglichkeiten in einer Peer-Group sowie Zugang zu Netzwerken (im Sinne: Zugang zu männlichen und weiblichen Schlüsselpersonen) eine zentrale Rolle für den beruflichen Erfolg von Frauen spielen. Eine weitere Einsicht war, dass die Art und Weise, wie innovative, wirkungsvolle F\&I-Lösungen für komplexe Problemstellungen heutzutage entstehen (im Vergleich zum klassischen Wissensgenerierungsprozess) gewandelt hat: So besteht zunehmend die Notwendigkeit zur Zusammenarbeit von unterschiedlichen Akteurinnen und Akteuren im Innovationssystem in interdisziplinären Teams. Aber auch die Einbeziehung von potentiellen Anwenderinnen und Anwendern wie auch Stakeholdern bereits im Entwicklungsprozess von Lösungen, um die Komplexität von Forschungs- und Innovationsherausforderungen (z.B. im Bereich der Grand Challenges, 17 globalen Nachhaltigkeitsziele - SDGs) zu bewältigen, erweist sich zunehmend als zielführend. Dafür benötigt es auch entsprechende Innovationskompetenzen und Denkweisen.

Für das Design des neuen Formats ergab sich daraus einerseits, das neue Angebot in Richtung Stimulierung neuer Netzwerke, Peer-Group sowie zum Erlernen neuer Innovationswerkzeuge umzugestalten. Andererseits schien es wichtig, die Zielgruppe zu verbreitern: Anstatt hauptsächlich Frauen aus der klassischen Forschung und Wissenschaft bzw. Unternehmerinnen anzusprechen, sollten auch Innovatorinnen aus anderen Sektoren (z.B. im Bildungsbereich, in der Kreativwirtschaft, im Sozialen), die an der Schnittstelle zu Forschung \& Technologie arbeiten, ein bezogen werden.

\footnotetext{
${ }^{2}$ wp eval_applied-sciences_final (convelop.com)

${ }^{3}$ Begleitevaluierung der Pilotausschreibung Ideen Lab 4.0. Zusammenfassung. - fteval Repositorium
} 


\section{Durchführung des Pilot Innovatorinnen 6/2020-4/2021}

Im Frühjahr 2020 startete die FFG die Bewerbungsmöglichkeit für das w-fFORTE Innovatorinnen Leadership-Programm. Angesprochen wurden Forscherinnen und Praktikerinnen aus F\&l, die große Ideen mit Impact für Wirtschaft und Gesellschaft (z.B. mit Blick auf die Grand Challenges, SDGs) voranbringen wollten. So konnten z.B. ein neues Vorhaben oder Forschungsgebiet aufgesetzt, ein neues Aufgabengebiet oder eine neue Funktion angestrebt oder neue Kooperationen aufgebaut werden. Ebenso war es möglich, in ein neues Gebiet (Disziplin, Branche, Themenfeld, Tätigkeitsfeld) einzusteigen oder eine Innovation durch die Kombination von Erfahrungswissen (z.B. aus der Kreativwirtschaft, Social Entrepreneurship) mit Forschung \& Wissenschaft zu entwickeln.

Das in 3 Modulen aufgebaute, über 10 Monate laufende Programm zeichnete sich durch Empowerment-Aktivitäten und den Erwerb („Hands-on“ entlang einer Frühphasen-Idee) neuer Innovations- und Kooperationskompetenzen aus. Ziel war es, Klarheit für die individuelle "Mission Zukunft" zu bekommen, diese im Austausch mit anderen (z.B. in der Peer-Group, mit Mentorinnen und Mentoren, Reality-Check mit Anwenderinnen und Anwendern, Expertinnen und Experten) systematisch in Richtung erste Schritte voranzubringen und anderen verständlich zu machen.

Eingeladen, sich zu bewerben, waren Frauen aus der anwendungsorientierten oder kooperativen Forschung \& Entwicklung und Praktikerinnen (z.B. aus Kreativwirtschaft, Social Entrepreneurship, Unternehmen aller Branchen), die forschen oder in Kooperationen Innovationen entwickeln wollten. Interessentinnen mussten sich mit einem Motivationsschreiben bewerben, in welchem sie Fragen zur Person und einer Frühphasen F\&I-Idee (mit der sie sich während des mehrmonatigen Programms auseinandersetzen wollten) beantworteten.

Trotz des zeitgleich ersten Lockdowns und des vollkommen überraschenden und sehr herausfordernden COVID-Pandemieumfelds bewarben sich 55 Frauen. Eine Jury wählte daraus 21 Teilnehmerinnen. Im Juni 2020 startete dann die nach unterschiedlichen Kriterien (z.B. Organisationsund Erfahrungshintergrund, Disziplin (von Digitalisierung, Energie- \& Mobilitätswende, Nachhaltigkeit über Gesundheits- \& Pflegewissenschaften bis hin zu gesellschaftlichen Innovationen) - bunt zusammengesetzte Gruppe in eine gemeinsame Lern- und Gestaltungsreise: 43\% der Teilnehmerinnen waren zwischen 25-34 Jahre alt, 38\% zwischen 35-44 Jahre, 14\% zwischen 45-55 Jahre. Rund zwei Drittel der Frauen stammten aus der F\&E-Community; darüber hinaus kamen Teilnehmerinnen z.B. aus dem Social Entrepreneurship-Bereich oder Innovationsmanagement. Aufgrund des Pandemie-Umfelds wurde der Durchgang online durchgeführt. Für die aus fünf verschiedenen Bundesländern kommenden Frauen entfiel somit die Anreise nach Wien.

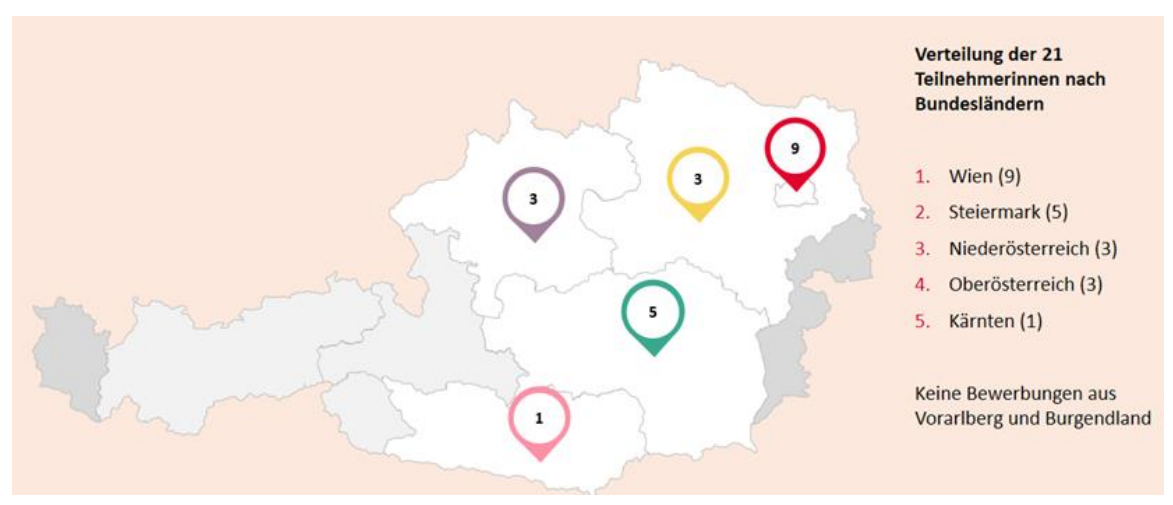

Das Leadership-Programm wurde inhaltlich von der FFG geleitet, die Empowerment-Aktivitäten wurden u.a. mit externen Trainerinnen und Trainern umgesetzt. Die 3 Module enthielten einen Methoden-Mix aus Coaching, Gruppendynamik, angewandter Improvisation sowie Design Thinking, kreativer Problemlösung, Co-Creation, LEGO@ Serious Play@ und Visual Thinking. Zusätzlich gab es: 
Webinare und Coachings zur Ideenkonkretisierung, Expertinnen-/Experten-Feedback (Co-Creation, Ideenpitch), ein Kamingespräch sowie moderierte Peer-Group-Treffen.

Angesichts der positiven Ergebnisse der begleitenden Erhebung und des kommunizierten weiterführenden Engagements der teilnehmenden Frauen wurde nach dem Programmende der Aufbau eines Alumnae-Netzwerks gestartet. Auf Basis der Evidenzen aus der begleitenden Erhebung arbeitet die FFG in Zusammenarbeit mit dem BMDW an einer Ausrollung weiterer Aktivitäten für Gestalterinnen in F\&l, die in Zukunft unter der Dachmarke INNOVATORINNEN durchgeführt werden.

Der Pilot hat inzwischen auch internationale Sichtbarkeit bekommen. Als Format, das „Kompetenzen für Openness und eine Kultur des Ermöglichens" bestärkt, ist es eines von 15 Best-Practice Beispielen in einer Studie des Stifterverband für die Deutsche Wissenschaft ${ }^{4}$. Die Studie zeigt Maßnahmen auf, welche die gezielte strategische Öffnung von Wissenschaft und Innovation und dadurch die Wirkungspotenziale für Wirtschaft, Wissenschaft und Gesellschaft vorantreiben. W-fFORTE Innovatorinnen stärkt damit auch die österreichische Open Innovation-Strategie.

\section{Evidenzen aus der Begleiterhebung: Was macht w-fFORTE Innovatorinnen aus?}

Die Begleiterhebung startete im Herbst 2020 und umfasste zwei Online-Befragungen der Innovatorinnen zu den jeweiligen Schritten bzw. Modulen in der Lern- und Gestaltungsreise sowie einen abschließenden Reflexions-Workshop gemeinsam mit der FFG und dem BMDW im April 2021. Alle Innovatorinnen haben die begleitende Erhebung umfangreich unterstützt und dadurch maßgeblich zur Qualitätssicherung und Weiterentwicklung des Pilots beigetragen. Die hohe Wertschätzung der Innovatorinnen gegenüber der FFG für ihr außerordentliches Engagement und die professionelle Abwicklung sind als besonders hervorzuheben.

Ausgangspunkt der begleitenden Erhebung war die Frage, welche Kompetenzen und Fähigkeiten braucht es, um als Innovatorin eine gestaltende Rolle einzunehmen, um auch den Pilot bestmöglich absolvieren zu können? Und zugleich, wie schätzen sich die Pilot-Teilnehmerinnen selbst ein, wie beurteilen sie ihre eigenen Fähigkeiten und Kompetenzen? Infolge ist auch das sogenannte „Empowerment-Rad“ entstanden.

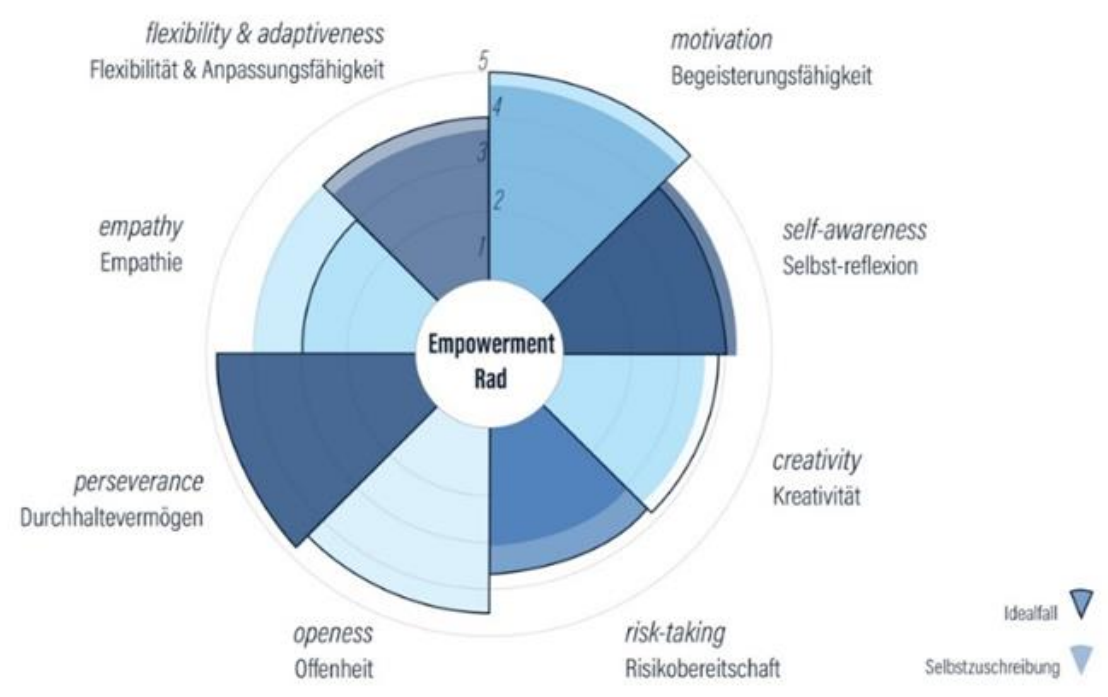

${ }^{4}$ Openness in internationaler Wissenschafts- und Innovationspolitik - Was Deutschland lernen kann (Juni 2021) 
Es zeigte sich, dass es eine hohe Übereinstimmung zwischen den Kompetenzen und Fähigkeiten, die als notwendig angesehen wurden, um den Pilot-Durchgang bestmöglich zu absolvieren, und der eigenen Selbsteinschätzung gab. Die Motivation, das Durchhaltevermögen und die Offenheit wurden dabei als besonders wichtige Fähigkeiten angesehen.

Der Weg ist das Ziel: Ziel der Lern- und Gestaltungsreise ist es, innovative Ideen aufzugreifen und diese zu präzisieren bzw. zu konkretisieren, sowie diese in Richtung Vorhaben weiterzuentwickeln. Dabei können natürlich auch Ideen abgeändert, adaptiert oder gar verworfen werfen. Die begleitende Erhebung hat gezeigt, dass es bei 37\% (7 Innovatorinnen) tatsächlich zu einer Änderung der ursprünglichen Idee kam. Und es war insbesondere das Format "Co-Creation Workshop", welches maßgeblich dazu beitrug, die Innovatorinnen in ihrer Ideenkonkretisierung zu unterstützen.

Überhaupt wurden die seitens der FFG eingesetzten Formate durchwegs als sehr positiv wahrgenommen. Als von den Teilnehmerinnen besonders positiv wurden dabei die Formate "Workshops (begleitet durch externe Coaches)", das "Webinar für Ideenkonkretisierung", die "ZOOM-Freiräume und die Begleitung für die Ideenkonkretisierung durch die FFG", sowie der "CoCreation Workshop" bewertet. Festgehalten von den Teilnehmerinnen wurde auch, dass gerade die Mischung und Vielfalt an Formaten den Pilot w-fFORTE Innovatorinnen als „besonders" auszeichnete.

Die Lern- und Gestaltungsreise, ein Weg durch viele unterschiedliche Formate, wurde von allen Teilnehmerinnen für die Umsetzung ihres Ziels als wichtig und relevant angesehen. Als besonders inspirierend wurden dabei die Formate mit LEGO@ Serious Play@ und "Mission Road Map" wahrgenommen. Beide Formate ermöglichten es, eigene Denkmuster zu erweitern, Zeit für Reflexion und Visualisierung zu schaffen, aber auch eigene Ansichten und Perspektiven zu hinterfragen. Als maßgeschneidert, an die individuellen Bedürfnisse gut abgestimmt, wurde das „Umsetzungscoaching“ wahrgenommen, während das „Empowerment Coaching“ insbesondere Kraft vermittelte. Das Format "Meine Mission -Mein Auftritt“ unterstützte schließlich den persönlichen Auftritt der Innovatorinnen und damit das Können "das eigene Vorhaben nach außen zu vermitteln". Allen im Rahmen von w-fFORTE Innovatorinnen vermittelten Formaten wurde ein hoher Nutzen zugeschrieben.

Eine weitere Frage der Begleiterhebung beschäftigte sich damit, ob sich durch die Lern- und Gestaltungsreise auch die Sichtweisen bzw. das Mindset verändert haben, oder es gar auch möglich war, die Persönlichkeit zu stärken?

Tatsächlich gaben viele der Innovatorinnen an, dass sich durch die Lern- und Gestaltungsreise ihre persönliche Sichtweise/ihr Mindset geändert hat; das heißt, dass es v.a. zu einem breiteren Blick auf die eigene Idee kam. Zudem sind die Innovatorinnen sich ihrer eigenen Stärken bewusst geworden und haben dadurch auch mehr Selbstbewusstsein erlangt. Die Innovatorinnen sind damit auch selbstbestimmter im Arbeitsumfeld aufgetreten. Auch haben die Innovatorinnen beobachtet, dass sie einen offeneren Zugang zu anderen lernten, damit ihre eigenen Ideen diskutieren, neue Inputs und Tools ausprobieren konnten. Durch die Lern- und Gestaltungsreise, gaben die Innovatorinnen an, sich dann auch auf die wesentlichen Punkte der Idee fokussiert zu haben. Insgesamt kam es damit zum Kennenlernen von neuen Arbeitsweisen und Tools, wie auch hat sich bei vielen Innovatorinnen der Blick auf sich selbst verändert.

Viele der durch den Pilot w-fFORTE Innovatorinnen erworbenen Fähigkeiten und Kompetenzen wurden von den Innovatorinnen auch bereits in der Praxis angewandt. Die Anwendung fand dabei in unterschiedlichen Settings und Situationen statt, wie z.B.:

- Bewerbungsprozess, wo das Vertrauen in eigene Skills gestärkt war, Visualisierungs-Skills genutzt wurden und dadurch eine bessere Positionierung erreicht wurde; 
- Auftreten vor potentiellen Projektpartne rinnen und -partnern, welches mitunter zur Akquise (mit Erfolg) von drittmittelfinanzierten Projekten auf nationaler und internationaler Ebene via interdisziplinäre Teams führte;

- Vernetzung, welche durch persönliche Präsentation und Weiterentwicklung unterstützt wurde;

- Projektentwicklung, die durch neue Ideen und Sichtweisen aufgegriffen wurde;

- Methodeneinsatz, wo Erlerntes aus Workshops in der Praxis in der Projektumsetzung Anwendung fand;

Haben Sie die Kenntnisse, entwickelten Fähigkeiten/Kompetenzen aus w-fFORTE bereits in der Praxis angewendet (wie z.B. im Berufs-leben)?

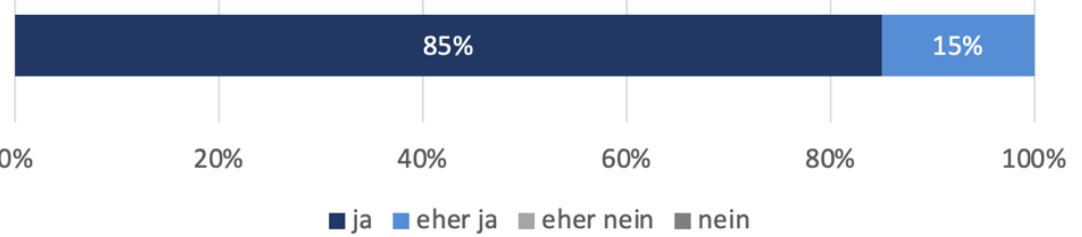

Eine besondere Rolle bzw. Funktion auf dem Weg, in der Lern- und Gestaltungsreise, nahm für die Innovatorinnen die Peer-Group ein. Die Peer-Group unterstützte die Innovatorinnen vor allem in der Vernetzung, Motivation und in emotionaler Hinsicht. Als besonders wertvoll wurden von den Innovatorinnen dabei auch die neuen Blickwinkel, die Anregungen und das ehrliche Feedback von außen wahrgenommen. Ebenso wurde die große Diversität der Teilnehmerinnen für die Lern- und Gestaltungsreise als förderlich angesehen.

\section{Das Besondere an w-fFORTE Innovatorinnen}

Als die am häufigsten genannten positiven Aspekte des Pilot-Durchgangs wurden die gegenseitige Unterstützung und die Wertschätzung genannt. Insbesondere der Austausch unter den Frauen wird als großer Mehrwert gesehen. In dem Kontext nimmt auch die Peer-Group eine bedeutsame Rolle ein. Viele Innovatorinnen nahmen in der Peer-Group die Chance wahr, sich zu öffnen, zu reflektieren, zu diskutieren, wertschätzenden Input von außen einzuholen und voneinander zu lernen. Die PeerGroup reichte zudem über die Formate von w-fFORTE Innovatorinnen hinaus; tatsächlich nahmen viele Innovatorinnen an einem selbst organisierten Peer-Austausch außerhalb der Formate von wfFORTE teil.

Insgesamt wurden die Innovatorinnen be- und gestärkt, ihre eigenen Ideen aufzugreifen, diese zu konkretisieren und weiterzuentwickeln. Der Weg zur gestaltenden Rolle als Innovatorin durch die Lern- und Gestaltungsreise ist gelungen.

Dieser Erfolg wird unterstrichen durch die Evidenz, dass die Innovatorinnen sogar den durch COVID erschwerten Rahmenbedingungen trotzten. Gerade die Durchführung der Formate im Online-Modus ermöglichte es, dass die Innovatorinnen trotz vieler weiterer Verpflichtungen (Beruf, Familie usw.) am Programm teilnehmen konnten. Damit unterstützte w-fFORTE Innovatorinnen auch die Vereinbarkeit von Beruf, Weiterbildung bzw. persönlicher Weiterentwicklung und Familie. Dennoch ist ein persönliches Kennenlernen und Treffen ein Wunsch für die zukünftigen Innovatorinnen. 


\section{Der Impact von w-fFORTE-Innovatorinnen}

Die Begleiterhebung zeigt eindeutig, dass der Pilot w-fFORTE Innovatorinnen Frauen in vielen karriere- und F\&E-relevanten Fähigkeiten und Kompetenzen unterstützt hat. Das Empowerment erfolgt durch unterschiedliche, gut aufeinander abgestimmte Formate der Lern- und Gestaltungsreise. Die Innovatorinnen haben dabei vor allem gelernt, „groß zu denken“ und dadurch Optionen zu erkennen und zu ergreifen. Die neuen Ansichten und Denkweisen wurden auch bereits während der Teilnahme am Pilot-Durchgang in der beruflichen Praxis erprobt und eingesetzt - mit beachtlichem Erfolg. Der Erfolg reicht dabei von einer interdisziplinären Projektkonzeption und erfolgreichen Drittmitteleinwerbung auf nationaler und EU-Ebene bis hin zur Positionierung in der Führungsebene. MINT-Karrieren wurden nicht verlassen, stattdessen wurden sie durch w-fFORTE Innovatorinnen motiviert weiterverfolgt. Des Weiteren kam es während der Teilnahme am Programm zu einer Cross-Industry-Kooperation, konkret zu einer Kooperation mit einem Forschungs-Startup, wodurch ein neues Geschäftsfeld entstand und ein Planungstool für Intensivstationen entwickelt wurde; das Tool wird mittlerweile in 17 Ländern eingesetzt.

Ganz im Sinne der F\&I-Mission wurden Kontakte und Netzwerke auf- und ausgebaut. Die Peer-Group nahm dabei wie bereits zuvor erwähnt eine besondere Rolle ein, sie wirkte bestärkend und zugleich motivierend.

W-fFORTE Innovatorinnen bietet damit einen großen Mehrwert. Die auf langer Vorarbeit basierende und durch viel Engagement geprägte Programmkonzeption bestärkt und unterstützt Frauen, ihre Fähigkeiten und Kompetenzen weiter auszubauen und durch die Lern- und Gestaltungsreise innovative Ideen aufzugreifen und diese in Richtung Umsetzung weiterzuentwickeln. Frauen in F\&I können damit eine gestaltende Rolle einnehmen und werden damit auch sichtbar. Der Plan, die PeerGroup in einem Alumnae-Netzwerk weiterzuführen, soll diesen Erfolg verstetigen.

\section{KOMMENTARE VON TEILNEHMERINNEN}

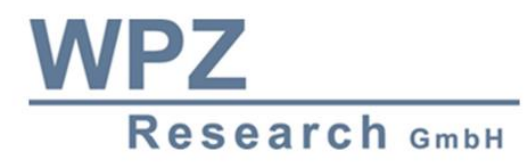

\begin{abstract}
Der gemeinsame Austausch sowie das Arbeiten an sich selbst und der eigenen Vision mit großartigen Kolleginnen ist unglaublich wertvoll. Gerade in diesem Jahr war der Austausch mit den Kolleginnen ein wichtiger und sehr vertrauter Anker.
\end{abstract}

Der Mehrwert des Programms liegt darin, dass es interdisziplinär ausgerichtet ist, das weitet nicht nur den Blick und führt zu anderen Ideen, sondern ist auch hilfreich, um auf Expertinnen zurückgreifen zu können für Fragen, die das eigene Fachgebiet übersteigen; dass es ein Frauennetzwerk ist (das erleichtert viel).

Ich bin persönlich gewachsen, bin besser in Verhandlungs- und Bewerbungsgesprächen, bei der Projektentwicklung/Akquise, beim Auftreten vor potenziellen Projektpartner*innen und habe neue Angebote angenommen
Durch das Programm konnte ich besser netzwerken, indem ich Netzwerkkontakte an w-fFORTE Mitglieder vermittelt habe und Innovatorinnen in anderen Netzwerken als Expertin genannt habe und auch für eine gemeinsame Veranstaltung zusammengearbeitet habe.

Programmmanagement war top!

Branchenübergreifend und damit sehr anregend und trotzdem eint Innovatorinnen und Trainer*innen: Neugierde, Macherqualitäten, Durchhaltevermögen, eine positive Haltung gegenüber dem Leben und das Einstehen für Diversity und Gendergerechtigkeit. 


\section{Zur zukünftigen Relevanz von Formaten wie INNOVATORINNEN}

Frauen handeln verantwortungsvoll. Das F\&I-Potential von Frauen ist systemrelevant. Die Förderung von Innovatorinnen ist nicht nur systemrelevant, es ist eine gesellschaftspolitische Aufgabe.

Gerade in Zeiten des Umbruchs ist es erforderlich, neue Ideen mit potentiellem Markterfolg und Wirkung für die Gesellschaft aufzugreifen und diese in Richtung Umsetzung zu führen. Dies gelingt durch Empowerment, durch den Einsatz von strategisch klug abgestimmten und eingesetzten Formaten und durch Persönlichkeitsentwicklung. Ein professionelles Programmmanagement wie auch die Möglichkeit, Teil einer Peer-Group zu sein, sind zusätzliche wesentliche Erfolgsfaktoren.

Für die Fortführung des Empowerments gibt es nun für die Zukunft zwei grundsätzliche Überlegungen. Diese sind:

1. Angesichts des großen Erfolgs, den Pilot-Durchgang w-fFORTE Innovatorinnen als eigenes Frauenförderprogramm weiterzuführen. Beweggründe hierfür sind: Frauen benötigen einen geschützten Raum - nur 7,7\% von FFG-geförderten Unternehmen werden von Frauen geführt $^{5}$ (Dies ist u.a. als Beleg zu sehen, dass es „Frauen in F\&E nach wie vor schwer haben, in Führungspositionen zu gelangen“) sowie das auf Bundesebene kommunizierte Ziel, "den Anteil von Frauen an Forscherinnen und Forschern zu steigern" (Zahlreiche einschlägige Studien, u.a. der jährlich erscheinende Österreichische Forschungs- und Technologiebericht ${ }^{6}$, zeigen diesen Handlungsbedarf seit Jahren auf; Österreich hat hier gerade auch im internationalen Vergleich einen dringenden Aufholbedarf).

2. Angesichts der Suche nach neuen Wegen bzw. Formaten und der hohen Professionalität der FFG (Beleg: $100 \%$ Zufriedenheit seitens der w-fFORTE-Innovatorinnen mit der Programmabwicklung und darüber hinaus herausragendes Lob für die Programmleitung) werden Überlegungen angestellt, wie die positiven Erfahrungen und die Expertise des Pilotdurchgangs w-fFORTE Innovatorinnen für das Programmportfolio der FFG genutzt werden können; mitunter ist dabei zu überlegen, welche (bekannten) Dreh- und Angelpunkte für eine systemische Hebelwirkung durch die Nutzung der Expertise herangezogen werden können.

\footnotetext{
${ }^{5}$ Siehe Keuschnigg, C., Gogola, G., Johs, J., Kritzinger, M., Sardadvar, S. (2020): Wirkung von Forschungsausgaben, Studie im A uftrag des BMDW, St. Gallen - Wien.

${ }^{6}$ Österreichischer Forschungs- und Technologiebericht 2021
} 